\title{
Choana Morphogenesis in the Post Hatching Developmental Stages of Muscovy Ducks
}

\author{
Fatma A. Madkour ${ }^{1}$, Kamal Abdalla ${ }^{2}$ and Salma Mohamed ${ }^{1}$ \\ ${ }^{1}$ Department of Anatomy and Embryology, Faculty of Veterinary Medicine, South Valley \\ University, Qena 83523, Egypt \\ ${ }^{2}$ Department of Anatomy and Histology, Faculty of Veterinary Medicine, Assiut University
}

\section{Abstract}

This study was performed by using Fourty nine Muscovy ducks to obtain enough information about the development of the choanal slit from 1 to 60 days old. The choanal slit formed nearly one-third of the total length of the palate in all studied ages; consisted of rostral narrow and caudal wide parts. The ratio of the length of the narrow part to the total length of the choanal slit was generally low at the young ducks and increased in the older ones, while that of the wide part reversing the ratio of the narrow part. By the SEM, 2 rows of papillae could be identified on the edges of the wide part of the choanal slit in all studied ages. The papillae of those rows were mostly cone-shaped with pointed apices, but some of them were dome-shaped with blunt apices could be demonstrated at 60 days old. Numerous openings of the intraepithelial glands were scattered within the epithelium of the choanal slit. At the edges of the choanal slit at one day old duck, the stratified squamous epithelium continued for a short distance before transforming into the ciliated respiratory epithelium. This epithelium was interrupted by intraepithelial mucous glands. These glands and their ducts surrounded by multiple lymphatic infiltration and nodules at 15- 60 days old. The lamina propria of the palate at the level of the choanal slit contained palatine salivary glands, these glands in older ages surrounded by numerous lymphatic nodules.

Keywords: Choanal slit, Ducks, SEM, Papillae.

DOI: $10.21608 / \mathrm{svu} .2019 .11860 .1009$

Received: April 12, $2019 \quad$ Accepted: May 11, $2019 \quad$ Published: June 6, 2019

*Corresponding Author: Fatma A. Madkour E-mail: madkour.f@vet.svu.edu.eg

Citation: Choana Morphogenesis in the Post Hatching Developmental Stages of Muscovy Ducks. SVU-IJVS, : 2 (2): 13-26, 2019

Copyright: ( ) Madkour et al., This is an open access article distributed under the terms of the creative common attribution license, which permits unrestricted use, distribution and reproduction in any medium provided the original author and source are created.

Competing interest: The authors have declared that no competing interest exists.

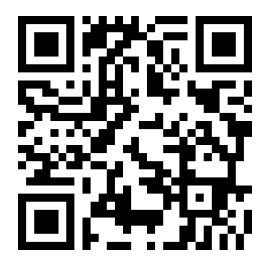




\section{Introduction}

All domestic ducks are obtained from the mallard, which distributed in Europe and North America. The Muscovy duck is the only species derived from wild ducks of central and South America (Stastny, 1985). The most important domestic birds after fowl are ducks (Nickel et al, 1977). Generally, the structural variations of the avian oropharyngeal cavity anatomy may affect in nutrition, food intake, and ingestion of the domestic birds so they have different feeding habits (Jayachitra et al., 2015). In most domestic birds, the size of choanal slit depends on the size and shape of the beak (Nickel et. al, 1977). The choanal slit is a median slit in the oral roof connecting the oropharynx to the nasal cavity consist of rostral narrow and caudal wide part (King and McLelland, 1984). The narrow rostral part may correspond to the median palatine suture of the mammals (Heidrich, 1908), while the caudal wide part is corresponding to the mammalian choana (Lucas and Stettenheim, 1972). The choanal slit is separated caudally from the infundibular slit by a mucosal bridge as in chicken and geese but closely related to the infundibular slit in the pigeon (Mohamed and Zayed, 2003). On each side of the choana of ducks (Anatidae) present a longitudinal ridge close to the palatine bones (McLelland, 1979). The mucous membrane of the borders of the choanal slit increased in thickness forming permanent mucosal folds (Hodges, 1974). The ventral aspect of the vomer can be observed within choanal slit in most the domestic birds except pigeon (Koch, 1973). In some birds, especially aquatic birds the position of the choanal slit located at the same level of the laryngeal opening to facilitate the direction of the inhaled air toward the respiratory passage (Hassouna, 2002). The development of the choanal slit is not available in the previous literature of many species of birds. So, this study was carried out to explain the gross, scanning and light microscopy of the choanal slit from 1 to 60 days old Muscovy ducks.

\section{Materials and methods}

This word was contrived fourty nine healthy Muscovy ducks (Cairina moschata) from local farms in Assiut Governorate. They were divided into 5 developmental stages $(1,7,15,30$ and 60 days old). After sacrificing and complete bleeding, the bird's heads were cut off. Five birds of each group were hired for the macro- anatomy examination. The oropharyngeal roof was dissected, washing under running tap water for one hour then fixed in $10 \%$ formalin and finally the morphological features and biometrical parameters of the choanal slit were recorded for each bird separately by using magnification lens and Precision Digital Vernier Caliper, and photographs were taken by using Panasonic Digital Camera. All measurements of this work analyzed by Statistical Package for Social Science (SPSS) software program, version 17.0 (Argyrous, 2005), the mean value \pm standard error ( $S E$ ) was used. For SEM, the choanal slit of 3 birds of only 1, 15- and 60days old groups were washed in normal saline and acetic acid 2\%, and then fixed in $4 \%$ glutaraldehyde solution for 24 hours. After fixation and dehydration, the specimens mounted on metal stubs then coated with gold palladium in the sputtering device then examined and photographed using JSM-4500 LV scanning electron microscope operated at $20 \mathrm{KV}$. For light microscopy manifestation, cross, sagittal and longitudinal sections from choanal slit were taken from 3 birds of each age, washed then fixed in $10 \%$ neutral buffer formalin. The samples were kept in $10 \%$ solution of EDTA in phosphate buffer (ph 7.4) for the process of decalcification. To ensure of good decalcification of the bony and cartilaginous contents of specimens, they were tested physically under finger touch, in general, the older ages require longer time in EDTA solution up to two months. After proper decalcification, the specimens washed at least from 6 to $12 \mathrm{hrs}$ under running tap water after that dehydrated in ascending grades of alcohol. Then they were cleared in methyl benzoate and 
embedded in paraffin wax. Sections of $5 \mu$ thickness were taken and stained with Harri's Hematoxylin and Eosin (H\&E) and Crossmon's Trichrome stains. The sections were examined with a light microscope. The stain techniques were reviewed by

(Bancroft and Gamble, 2008).

\section{Results}

The choanal slit was a median cleft located in the caudal part of the palate rostral to the level of the angle of the mouth by a variable distance depending on the age of the bird. This distance was very short $1.64 \mathrm{~mm}$ at one day old and increased with the advancement of the age to reach 8.16 $\mathrm{mm}$ at 60 days olds. Concerning the length of the choanal slit, the morphometrical study indicated that it was 8.08, 10.78, $13.12,20.26$ and $26.42 \mathrm{~mm}$ at $1,7,15,30$ and 60 days old respectively. It constituted a ratio from the total length of the palate in all examined ages ranged from $31.02 \%$ to $34.98 \%$. Consequently, in all ages the choanal slit formed nearly one-third of the total length of the palate. The choanal slit was situated nearly just rostral to infundibular cleft (Fig. 1A, B).
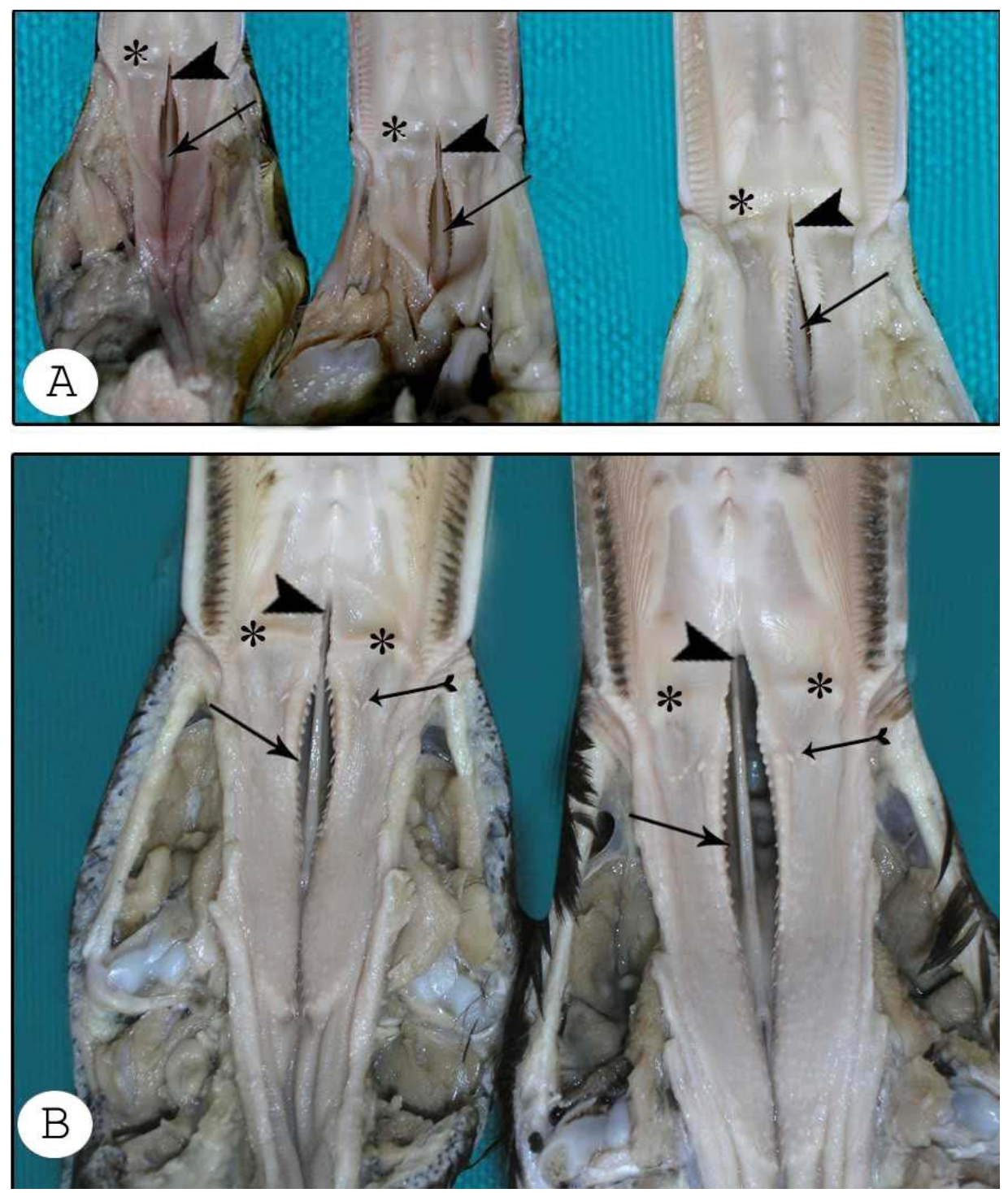

Fig. (1): Photograph of the choanal slit of 1,7and 15 days (A) and of 30and 60 days old ducks (B) from left to right, showing narrow part (arrow head), wide part (arrow) of choanal slit, a transverse row of caudally directed papillae (barbed arrow) and mucosal folds on each side of the narrow part (stars). 
The choanal slit consisted of two parts; narrow rostral and wide caudal (Fig. 1A, B). The position of these parts in relation to the laryngeal inlet, their lengths and their ratio to the total length of the choanal slit vary in different ages. In all examined ages, the narrow part of the slit located completely rostral to the level of the laryngeal inlet. But the relation between the position of the wide part and the laryngeal inlet was variable. About the caudal half of the wide part of the choanal slit was located opposite to the laryngeal inlet at 1-15 days old. However, nearly the wide part of the choanal slit lied opposite to the laryngeal inlet at 60 days old. With respect to the length of both the narrow and wide parts of the choanal slit, it was noticed that the narrow part measured $3.01 \mathrm{~mm}$ at one day old, then increased nearly one and half fold to become $4.16 \mathrm{~mm}$ at 15 days old. Furthermore, at 60 days old, it increased three and half folds to reach $10.76 \mathrm{~mm}$. The wide part of the choanal slit was $5.07 \mathrm{~mm}$ long at one day old, then increased nearly one and half folds to become $8.96 \mathrm{~mm}$ at 15 days old and nearly three folds to reach $15.66 \mathrm{~mm}$ at 60 days old. The relation between the length of each of the narrow and wide parts to the total length of the choanal slit clarified that the narrow part constituted $37.16 \%$ at one day old and $41.03 \%$ at 60 days old from the total length of the choanal slit. This ratio was generally low at the young ducks and increased in the older ducks. On the other hand, the ratio of the wide part to the total length of the choanal slit was $62.82 \%$ at one day old and decreased to become $58.96 \%$ at 60 days old. Consequently, this ratio was generally high in the young ducks, but decreased in the older ones. The width of the wide part of the choanal slit measured $1.07 \mathrm{~mm}$ at one day old; it developed with the advancement of the age to become $2.01 \mathrm{~mm}$ at 15 days old and reached its maximum value $4.67 \mathrm{~mm}$ at 60 days old. As a result, the width of the slit at 15 days old comprised about two folds and at 60 days old four folds when compared with that at one day old. The wide and narrow parts of the choanal slit were continuous with each other without any distinct line of demarcation except for a transverse row of caudally directed papillae was situated in both sides of the slit. In all developmental examined stages this row of papillae lied shortly behind the level of the angle of the mouth. The number and size of the papillae of the transverse row vary in the different ages. There were 2-3 fine papillae begin to appear at 1-7 days old, then 4 small sized caudally pointed papillae were identified at 15 days old. At 30-60 days old, the papillae became larger in size and widely spaced, the medially situated papillae were larger than the laterally situated ones (Fig.1A, B).

The edges of the choanal slit were generally thickened due to the presence of several papillae which differ in their number, size and arrangement basing on the age of the bird. At one day old, a longitudinal row of fine papillae was identified at the edge of the rostral $2 / 3$ of the wide part of the choanal slit. At 7 days old this row occupied the whole edges of the wide part of the slit. In addition, at this age another longitudinal row appeared medial to the previous row on the edges of the slit. Therefore, up to 7 days old, two longitudinal rows (medial and lateral) can be differentiated at the edges of the wide part of the choanal slit. At 15 days old, the papillae of the lateral row became larger than those of the medial row. Moreover, the papillae of the lateral row were pointed caudally, but those of the medial row were directed caudomedially. At 30-60 days the papillae of both rows became widely spaced. The medial row occupied the edges of the caudal half of the narrow part in addition to the edges of the whole wide part. The lateral row occupied the whole wide part, and only 2-3 small sized papillae from this row were observed on the edges of the narrow part. Moreover, at 60 days old about 4-5 very small pointed shaped papillae began to appear on the palate lateral to the choanal slit and opposite to the rostral half of its wide part. 
Table (1): The dimensions (in $\mathrm{mm}$ ) of the choanal slit

\begin{tabular}{|c|c|c|c|c|c|}
\hline & 1 day & 7 days & 15 days & 30 days & 60 days \\
\hline $\begin{array}{l}\text { Length of palate } \\
\text { Length of choanal slit } \\
\text { Ratio\% of choanal slit / } \\
\text { length of palate }\end{array}$ & $\begin{array}{l}25.36 \pm 1.16 \\
8.08 \pm 0.18 \\
31.86 \%\end{array}$ & $\begin{array}{c}30.81 \pm 1.04 \\
10.78 \pm 0.27 \\
34.98 \%\end{array}$ & $\begin{array}{c}41.29 \pm 0.79 \\
13.12 \pm 0.39 \\
31.18 \%\end{array}$ & $\begin{array}{c}64.27 \pm 2.15 \\
20.26 \pm 1 \\
31.52 \%\end{array}$ & $\begin{array}{c}82.96 \pm 4.65 \\
26.42 \pm 1.68 \\
31.84 \%\end{array}$ \\
\hline $\begin{array}{l}\quad \text { Choanal slit } \\
\text { Length of narrow part } \\
\text { Length of wide part } \\
\text { Ratio (\%) of narrow } \\
\text { part } \\
\text { Ratio (\%) of wide part } \\
\text { Width of wide part }\end{array}$ & $\begin{array}{c}3.01 \pm 0.11 \\
5.07 \pm 0.08 \\
37.16 \% \\
62.82 \% \\
1.07 \pm 0.06\end{array}$ & $\begin{array}{c}3.28 \pm 0.12 \\
7.50 \pm 0.25 \\
30.46 \% \\
69.52 \% \\
1.71 \pm 0.11\end{array}$ & $\begin{array}{c}4.16 \pm 0.12 \\
8.96 \pm 0.27 \\
31.72 \% \\
68.26 \% \\
2.01 \pm 0.06\end{array}$ & $\begin{array}{c}8.13 \pm 0.36 \\
12.12 \pm 0.65 \\
40.21 \% \\
59.78 \% \\
3.07 \pm 0.31\end{array}$ & $\begin{array}{c}10.76 \pm 0.46 \\
15.66 \pm 1.27 \\
41.03 \% \\
58.96 \% \\
4.67 \pm 0.54\end{array}$ \\
\hline $\begin{array}{l}\text { Distance between } \\
\text { choanal slit and angle of } \\
\text { mouth }\end{array}$ & $1.64 \pm 0.07$ & $2.28 \pm 0.12$ & $3.65 \pm 0.22$ & $5.96 \pm 0.25$ & $8.16 \pm 0.58$ \\
\hline
\end{tabular}

In general, at all developmental stages, the papillae of the edges of the choanal slit were larger rostrally and became smaller caudal wards. On both sides of the narrow part of the choanal slit a transverse mucosal elevation extending from the slit to the lateral edge of the palate was demonstrated in all examined ages. This elevation lied opposite to the level of the angle of the mouth (Fig.1A, B).

All statistical data of the choanal slit were shown in Table (1).

Concerning scanning electron microscopical findings of the narrow part of the choanal slit at all stages, it was observed that 2-3 primordia of papillae were demonstrated on the edges of the caudal portion of this part at one day old (Figs.2A \& 3A). At 15 days old, small-sized of narrow spaced papillae were detected on the edges of the caudal fourth of the narrow part of the choanal slit (Fig.2B). At 60 days old, the papillae became relatively larger and occupied the caudal half of the narrow part of the choanal slit (Fig.2C). The papillae on the edges of the wide part of the choanal slit were variable in shape, size and direction basing on the age of the bird; two rows 


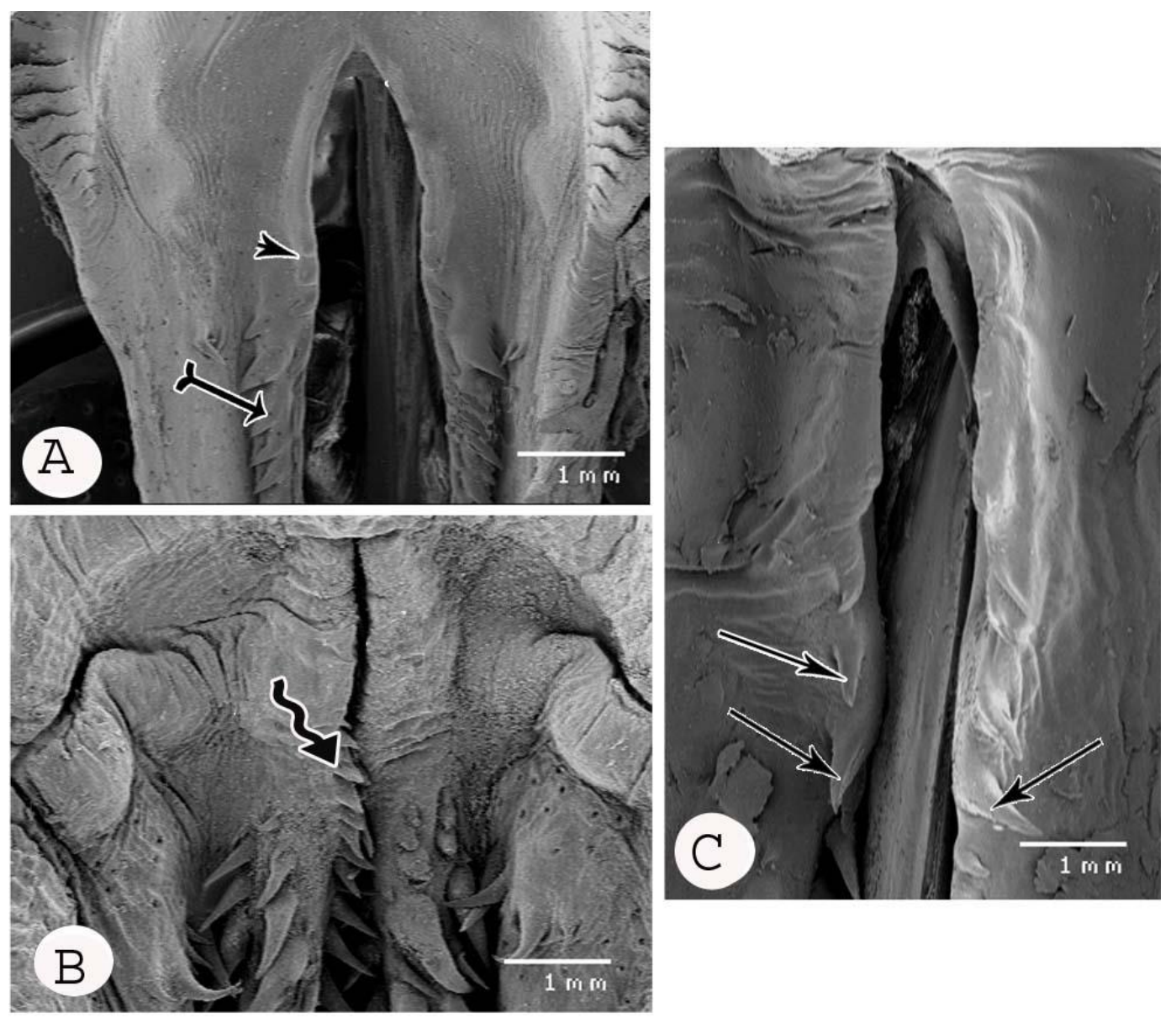

Fig. (2): Scanning electron micrograph of the narrow and rostral half of the wide parts of the choanal slit of one day (A), 15 days (B) and the narrow part of 60 days old ducks (C), showing the primordium of papillae on the edge of the caudal portion of the narrow part (arrow head), the medial and lateral rows of the small sized papillae on the edge of the wide part of the choanal slit (barbed arrow), small sized papillae on the edge of caudal 1/4 of narrow part of the slit (twisted arrow), large papillae on the caudal half of the narrow part of the choanal slit (arrows).

(medial and lateral) of small sized-papillae could be recognized on the edges of the wide part of the choanal slit at one day old. The papillae were generally cone-shaped directed caudolaterally. The papillae of the lateral row were larger than those of the medial row (Figs.3A). At 15 days old, the papillae of both rows were cone-shaped with pointed apices. The papillae of the lateral row were directed caudolaterally, while those of the medial row were directed caudomedially (Fig. 3C). At 60 days old, the papillae of both rows increased in size and were mostly cone-shaped with pointed apices, but some of them were dome-shaped with blunt apices (Fig.3E). In all studied ages, the papillae of the medial and lateral rows of the wide part of the choanal slit decreased in length caudal wards (Fig. 3A, $\mathrm{C}, \mathrm{D} \& \mathrm{E}$ ). Numerous openings of the palatine salivary glands were demonstrated at the roof of the palate on both sides of the choanal slit on all developmental stages (Fig.3B, D \&E). In addition, numerous openings of different shapes, sizes of intraepithelial glands were scattered within the epithelium of the choanal slit. At higher magnification, few fine cilia of epithelium were scattered between the openings of intraepithelial glands (Fig.4A, B).

By light microscopy, in cross sections at the level of the choanal slit of one day old, the covering epithelium was stratified squamous epithelium non cornified 

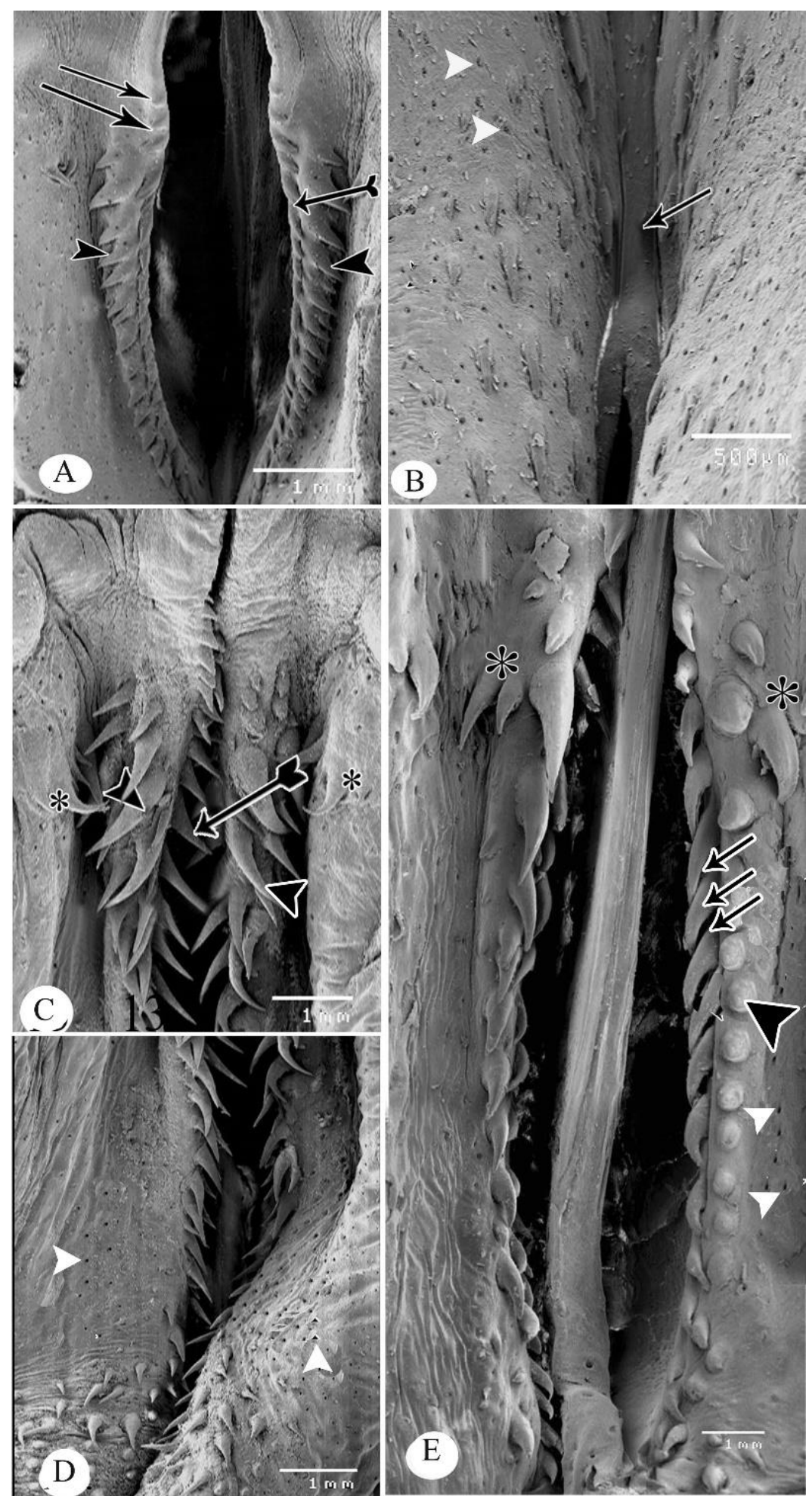

Fig. (3): Scanning electron micrograph of the wide part of choanal slit of one day (A, B), of 15 days $(C, D)$ and of 60 days old ducks $(E)$, showing 2-3 primordia of papillae on the caudal portion of the narrow part of the choanal slit (arrows), medial (barbed arrow) and lateral (arrow heads) rows of papillae on the wide part of the choanal slit, the junction between the choanal and infundibular slits (arrow) and openings of the palatine salivary glands (white arrow heads), two types of papillae; pointed apices (three arrows) and blunt apices (large arrow head), transverse row papillae between narrow and wide parts (stars). 

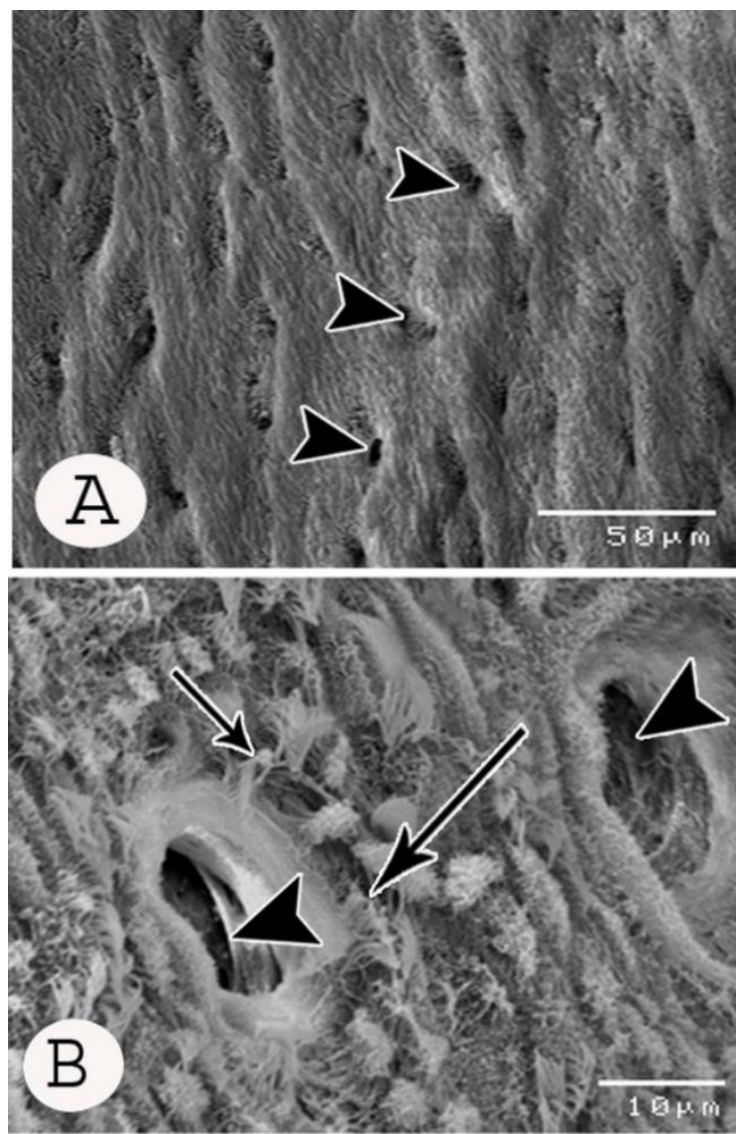

Fig. (4): Scanning electron micrograph of the epithelium of the choanal slit of one day old duck (A, B), showing numerous openings of the intraepithelial glands (arrow heads), fine cilia of epithelium (arrows) scattered between the openings of the intraepithelial glands.

Fig. (5): Photomicrograph of the lining epithelium of the choanal slit of one day old duck (A-C) stained with H\&E, showing stratified squamous epithelium of palate (arrow heads) transforming into respiratory epithelium (arrows) containing intraepithelial glands (GL) open on the surface of the epithelium, cilia of the respiratory epithelium (twisted arrow).

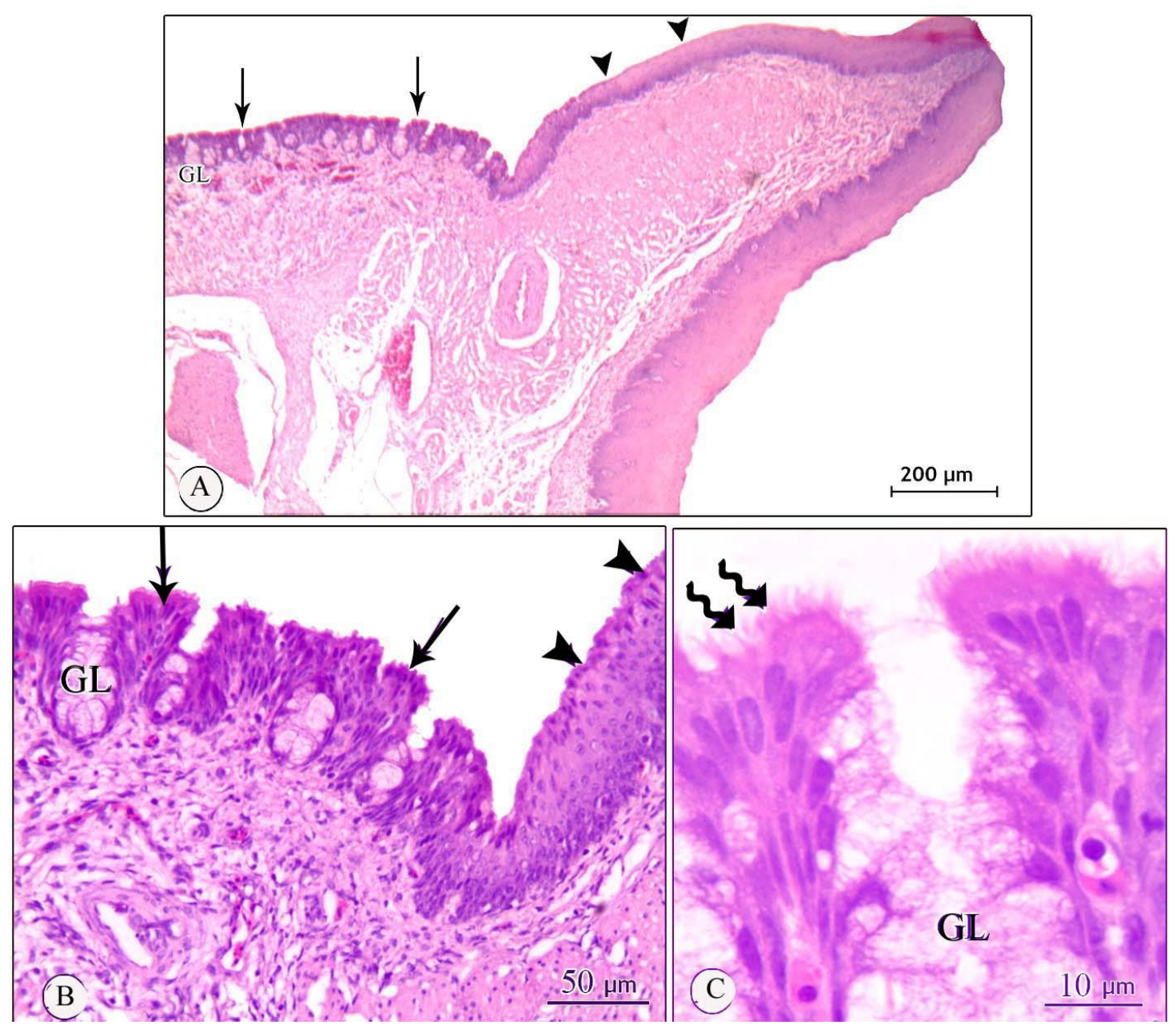




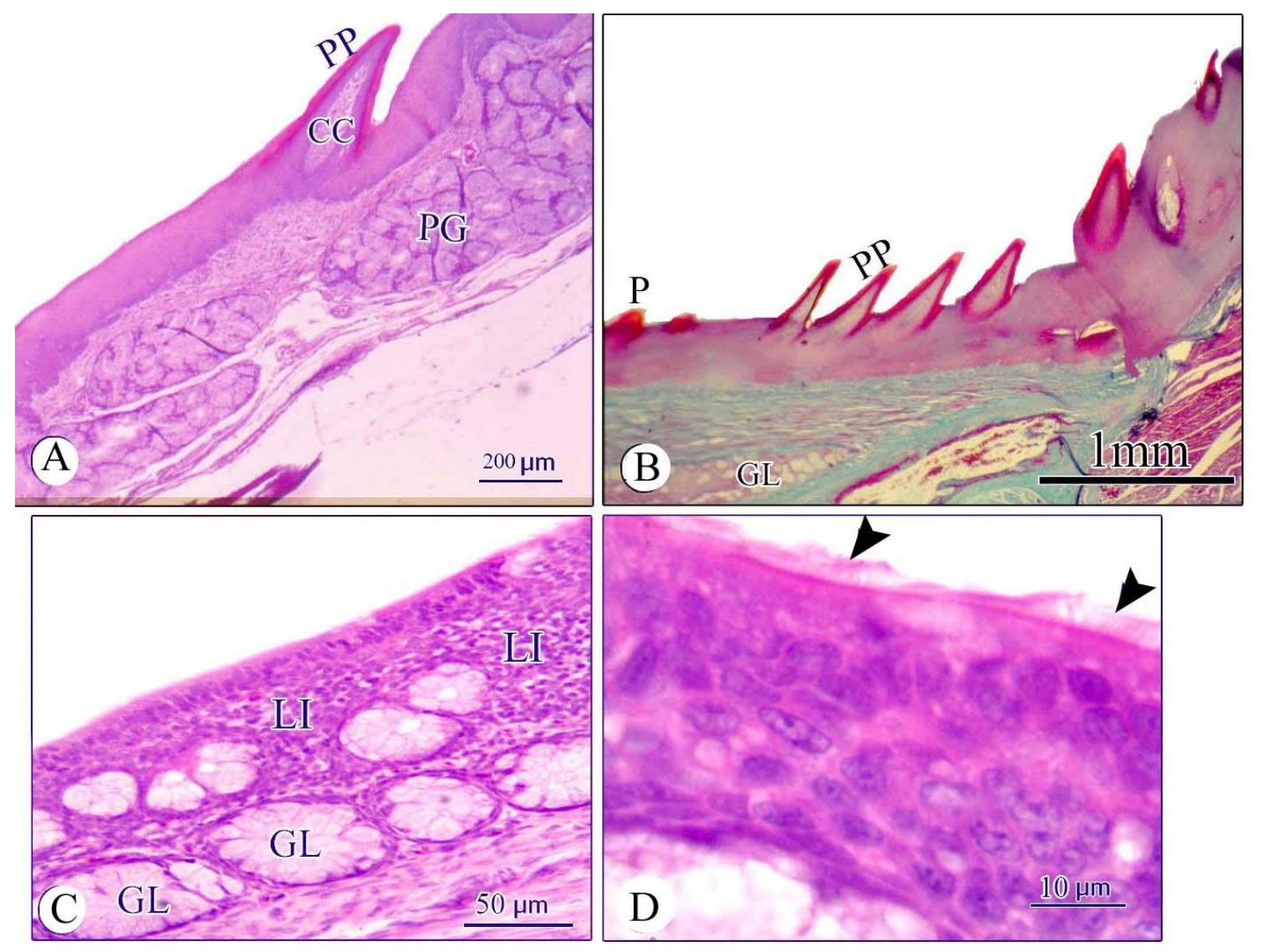

Fig. (6): Photomicrograph of the longitudinal section of the choanal slit of one day (A) and sagittal section of 7 days old ducks (B-C), (A, C\& D) stained with H\&E and (B) stained with Crossmon's Trichrome, showing keratinized papillae (PP), connective tissue core (CC), short papillae (p) at the narrow part of the slit, palatine glands (PG), intraepithelial glands (GL), Lymphatic infiltration (LI) and cilia of the epithelium (arrow heads).

continues for a short distance before transforming into pseudostratified columnar ciliated epithelium (ciliated respiratory epithelium) which was interrupted by intraepithelial mucous glands (Fig. 5A, B). Each gland was lined by a single layer of cuboidal to columnar cells containing foamy cytoplasm and oval basally situated nuclei, some of these glands open directly on the surface epithelium through a duct (Fig. 5B, C). At this age, no lymphatic infiltration was seen surrounding the intra epithelial glands. In sagittal sections, short papillae with a wide base and pointed apex appeared on both sides of the choanal slit at the level of its wide part (Fig.6A). The papillae were covered with less keratinized stratified squamous epithelium. The lamina propria contained palatine glands arranged in a longitudinal manner which were of a compound lobulated mucous type. Each gland was formed of glandular lobules, each lobule contained several secretory units lined with relatively low columnar cells with foamy vacuolated cytoplasm and oval basally situated nuclei close to the basement membrane. At 7-15 days old, in addition to the papillae of the wide part of the choanal slit another group of shorter papillae appeared at the level of the narrow part (Fig.6B). At these developmental periods, lymphatic infiltration was seen surrounding intraepithelial glands (Fig.6C, D). At 30-60 days old, the ciliated respiratory epithelium was well developed, and the intraepithelial glands became larger in size and the cytoplasm of the secretory cells were highly 
foamy. The intraepithelial glands and their ducts were surrounded by multiple lymphatic infiltration and nodules (Fig.7A). The papillae of the choanal slit became larger and highly cornified (Fig.7B). At these stages of development, the palatine glands became well developed and the lining secretory cells became tall columnar with highly foamy and more vacuolated cytoplasm. Moreover, these glands were surrounded by numerous lymphatic nodules (Figs.7C, D).

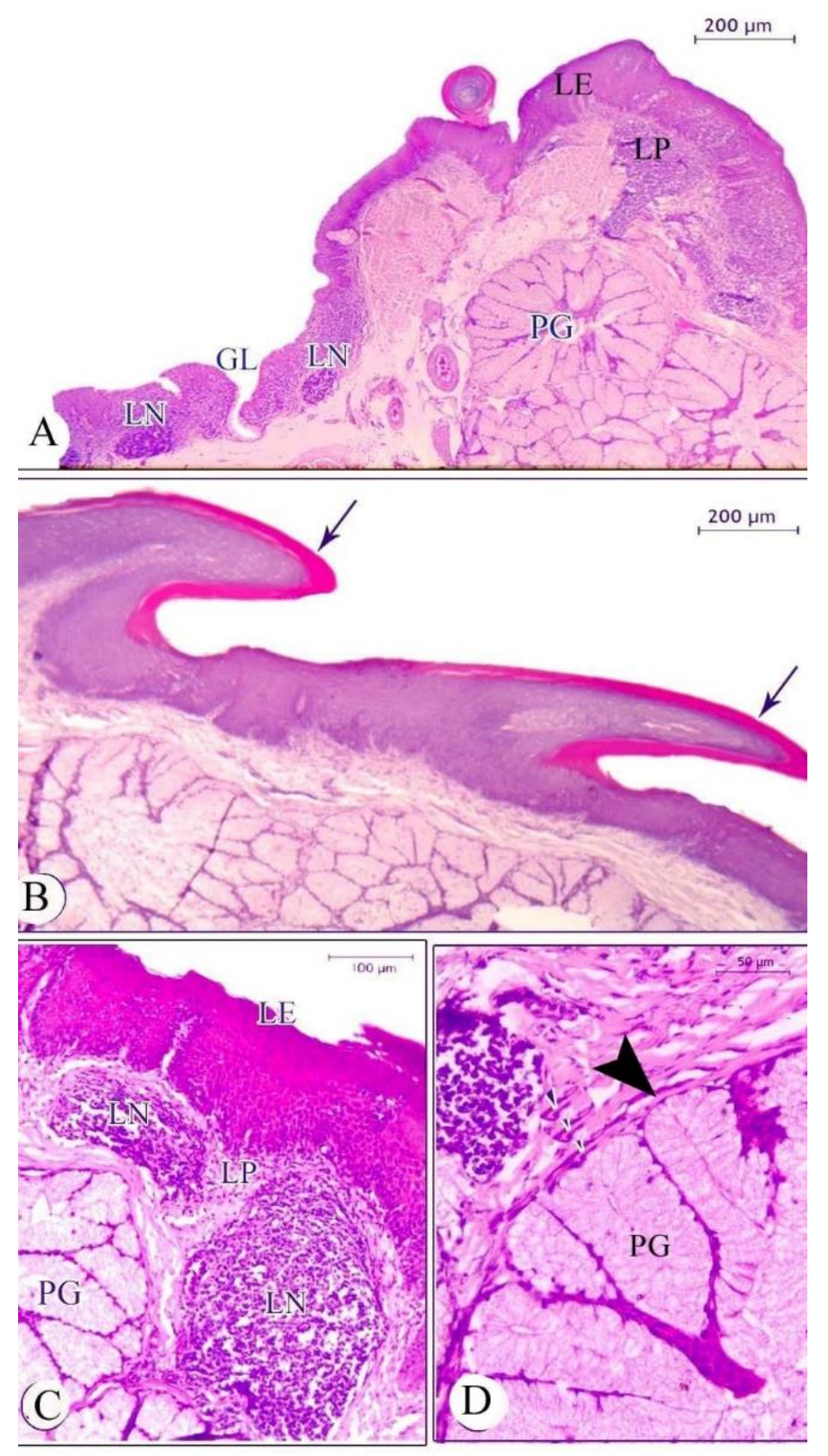

Fig. (7): Photomicrograph of the cross section (A, C \&D), of longitudinal section (B) of the choanal slit of 60 days old duck stained with H\&E, showing lamina epithelialis (LE) and lamina propria (LP) contained palatine salivary glands (PG) and lymphatic nodules (LN), intraepithelial glands (GL), larger and highly cornified papillae (arrows), secretory tubule of the palatine glands (PG) lined by tall columnar epithelium with highly foamy cytoplasm and flattened nuclei surrounded by connective tissue capsule (arrowhead). 


\section{Discussion}

It is observed that the choanal slit lies in the caudal part of the palate rostral to the level of the angle of the mouth by variable distances basing on the age of the duck. Corresponding to that observed in emu (Crole and Soley, 2010), in turkey (Sayed et al., 2017), in ostrich (Goppert, 1903; Porchescu, 2007; Tadjalli et al., 2008; Tivane et al., 2011) and in herons and ducks (Barge, 1937). However, the slit extends into the rostral part of the palate in the chicken and pigeon (Mohamed and Zayed,

2003). At this point, the choanal slit located completely within the pharyngeal cavity in duck (Hassouna, 2002). The present findings showed that the choanal slit in the examined ducks is situated nearly just rostral to infundibular cleft. Similar observation was reported in chicken (King and McLelland, 1984) and in pigeon (Mohamed and Zayed, 2003). In this respect, the two openings open together in the budgerigar (Evans, 1996; Dyce et al., 2002). In latter bird, the single median opening of the combined auditory tubes is in the middle of the choana (Evans, 1996). On contrast, the choanal slit is separated from the infundibular slit by $3 \mathrm{~mm}$ in the chicken and $6 \mathrm{~mm}$ in the goose (Mohamed and Zayed, 2003), and by few millimeters in chicken (Koch, 1973). In all examined ages, the choanal slit constitutes $31.02 \%$ to $34.98 \%$ from total length of the palate. Somewhat simulate the percentage in turkey which recorded $38.30 \%$ (Sayed et al., 2017). However, it constitutes 53.49\%, $50.0 \%$ and $27.06 \%$ of the total length of palate in the chicken, pigeon and goose respectively (Mohamed and Zayed, 2003). On other hand, the choanal slit constituted $49-50 \%$ in laughing dove and Japanese quail (Madkour, 2018).

The work under investigation revealed in all examined ages that the narrow part of the slit lies completely rostral to the level of the laryngeal inlet. About the caudal half of the wide part of the choanal slit is located opposite to the laryngeal inlet at 1-15 days old. However, the wide part of the slit lies opposite to the laryngeal inlet at 60 days old, like that recorded in duck (Hassouna, 2002), and in pigeon and goose (Mohamed and Zayed, 2003). During respiration the tongue closed the narrow part of the tongue, so the wide part facilitates the air to enter the larynx (Nickel et al., 1977). In all examined ages, on both sides of the narrow part of the choanal slit there is a transverse mucosal elevation. In African pied crow, mucosal elevations of the palate; the palatine ridges present on either side of the enlarged caudal part of the choana (Igwebuike and Eze, 2010). The statistical data indicated that, the wide part of the choanal slit in all examined ages constitutes $58.96 \%-62.82 \%$ of its total length. In this concern, the wide part constitutes about $39.1 \% ; 25.0 \%$ and $56.5 \%$ of the total length of the slit in the chicken, pigeon and goose, in turn the wide part is shorter than the narrow part in the chicken and pigeon, but slightly longer than it in the goose (Mohamed and Zayed, 2003). The wide part is double the narrow part in the duck (Hassouna, 2002). The wide part in the chicken is nearly half the narrow part, but in the duck and goose it is longer than the narrow part (McLelland, 1975). The short and caudally positioned choanal slit in the goose, compared with the chicken and the pigeon, may play role to prevent the entrance of water in the slit during sieving feed particles from the water (Mohamed and Zayed, 2003). This study clarified that a transverse row of papillae between 2 parts of the slit lies shortly behind the level of the angle of the mouth; a consists of 2-3 fine papillae at 1-7 days old, then 4 small sized caudally pointed papillae at 15 days old and the papillae become larger and the medially situated papillae are larger than the laterally situated ones in 30, 60 days old. In this connection, in turkey the transverse row of papillae lies opposite to the level of the angle of the mouth (Sayed et al., 2017). Madkour (2018) added that the line of 
separation between narrow and wide parts demarcated by caudolaterally directed papillae and mucosal elevation in laughing dove and by a transverse row of $\mathrm{V}$-shaped papillae in Japanese quail. With regard to the edges of the slit; by SEM 2 rows of papillae can be identified on the edges of the wide part of the choanal slit in all studied ages from 1-60 days old. The papillae of these rows are mostly cone-shaped with pointed apices, but some of them are domeshaped with blunt apices are demonstrated at 60 days old. Sayed et al., (2017) showed that the papillae of the edges of the narrow part are wedge-shaped and caudomedially directed and of the edges of the wide part are nearly situated in regular intervals.

Moreover, Mohamed and Zayed (2003) revealed that in goose the papillae on the edges of the choanal slit appear conical in shape with narrow bases encircled with concentric scales. However, in the chicken and pigeon they are tongue shape with wide bases. Functionally, the papillae which distributed around the choanal slit impede entrance the foods into the slit during swallowing and with that of the palate aiding in passage foods toward the esophagus.

The histological investigations revealed in all examined ages that the covering epithelium at the level of the choanal slit is stratified squamous epithelium non-keratinized transforming into pseudostratified columnar ciliated epithelium (ciliated respiratory epithelium) after short distance. In this connection, Mclelland (1975) and Mclelland (1979) mentioned that the stratified squamous epithelium of the palate is continuous with the epithelium of the nasal cavity at the nasal edge of the choanal slit. There is a general agreement that the oropharyngeal roof contains palatine salivary glands. The different classifications of these glands are described by several authors. In chicken, McLelland (1975); Nickel et al., (1977) and Samar et al. (1999) described medial and lateral groups of palatine salivary glands which extend on each side of the choanal slit. Madkour (2018) claimed that submucosa of the palate on each side of the choanal slit consisted of 2 groups of palatine salivary glands; medial and lateral groups in Japanese quail and few lobules of the medial salivary glands were observed in laughing dove. However, Mohamed and Zayed (2003) mentioned that the caudal part of the palate in the chicken and goose contains several openings of the palatine salivary glands, arranged in rows, on both sides of the choanal slit. While in the pigeon, few minute openings are demonstrated on both sides of the choanal slit at the junction of its narrow and wide parts. From a comparative study was applied upon the anatomical and histological investigations of 12 African black ostriches, 9 hens and 6 turkeys. It is observed that all the salivary glands are of a mucous structure and of a multilobular type (Porchescu, 2007). Gargiulo et al., (1991) and Samar et al., (1999) added these mucous secretions not only for nutrient ingestion but also for non-immune protection of the buccal cavity.

\section{Conclusion}

It turned out from the present study of the choanal slit of the post hatching Muscovy ducks aging from one to sixty days olds that the choanal slit formed nearly one-third of the total length of the palate. The Air passage rate was better in the older age than the young as result the caudal half of the wide part of the choanal slit was located opposite to the laryngeal inlet at 115 days old. However, nearly the wide part of the choanal slit lied opposite to the laryngeal inlet at 60 days old. With advancement of the age there were obvious increasing of the shape, number and size of the choanal papillae.

\section{References}

Argyrous G (2005). Statistics for research; with a guide to SPSS. Sage, London.

Bancroft JD and Gamble M (2008). Theory and practice of histological techniques,

Barge JAJ (1937). Kopfdarm. A. Mundhohle und ihre organe. I. 
Mundholendach und Gaumen In: Handbuch der vergleichenden Anatomie der Wirbelitere (L.Bolk, E.Goppert, E.W, Lubosch, eds). Berlin: urban and Schwarzenberg, 2948.

Crole M R and Soley JT (2010). Gross morphology of the intra-oral rhamphotheca, oropharynx and proximal esophagus of the emu (Dromaius novaehollandiae). Anatomia Histolpgia Embryologia, 112.

Dyce KM, Sack WO and Wensing CJG (2002). Textbook of Veterinary Anatomy, 3rd Ed. Saunders, An Imprint of Elsevier Science, Philadelphia, London, New York and Sydney.

Evans HE (1996). Anatomy of the budgerigar and other birds. In Diseases of cage and aviary birds edited by Rosskopf, W. J. and Woerpel, R. W., 3rdEd. A Lea \& Febiger, Williams \& Wilkins A wavely company Philadelphia.

Gargiulo AM, Lorvik S, Ceccarelli P and Pedini V (1991). Histological and histochemical studies on the chicken lingual glands. Br. Poult. Sci. 32, 693702.

Goppert E (1903). Die Bedeutung der Zunge fur den sekundae ren Gaumen und den Ductus nasopharyngeus. Morphol. Jahrb. 31, 311-359.

Hassouna EMA (2002). Morphological studies on the pharyngeal cavity of duck (Anas bousius domesticus). Assiut Vet. Med. J. 47(94), 21-41.

Heidrich K (1908). Die Mund und Schlundkopfhohle der Vogel und ihre Drusen, Morphol. Jahrb. 37, 10-69.

Hodges RD (1974). The Histology of the Fowl. Academic Press, London, New York and San Francisco.

Igwebuike UM and Eze UU (2010). Anatomy of the oropharynx and tongue of the African pied crow (Corvus albus). Vet. Arhiv 80 (4), 523-531.
Jayachitra S, Balasundaram K, Iniyah K, Sivagnanam $\mathrm{S}$ and Tamilselvan $\mathrm{S}$ (2015). Morphology of oropharyngeal cavity in guinea fowl (Numida meleagris). International Journal of Advanced Multidisciplinary Research IJAMR 2:99-102.

King AS and McLelland J (1984). Birds: Their Structure and Function, $2^{\text {nd }} E d$. Bailliere Tindall, London.

Koch T (1973): Anatomy of the Chicken and Domestic birds. The Iowa State University Press, Iowa.

Lucas AM and Stettenheim PR (1972). Avian Anatomy. Integument. Agriculture handbook 362. Department of agriculture, Washington, DC, GPO. Part II. Washington, United States department of Agriculture.579-592.

Madkour FA (2018). Morphological Studies of the Oral Roof of the Egyptian Laughing Dove (Streptopelia senegalensis aegyptiaca) and Japanese Quail (Coturnix japonicum). J. Vet. Anat. 11(2):17-39.

McLelland J (1975). Aves Digestive System: In Sission and Grossman's, The Anatomy of the Domestic Animals, Rev. R. Getty. Vol. 2, 5th Ed., W. B. Saunders Company, Philadelphia and London.

McLelland J (1979). Digestive system. In: Form and Function in Birds. (King, A.S., McLelland, J., Ed.). Academic Press, London, pp.69-181.

Mohamed SA and Zayed AE (2003). Gross anatomical and scanning electron microscopical studies on palate of some birds. Assuit Vet. Med. J. 49 (97), 1-17.

Nickel R, Schummer A and Seiferle E (1977). Anatomy of the domestic birds. Verlag Pawl Parey, Berlin and Hamburg.

Porchescu G (2007). Comparative morphology of the digestive tract of the black African ostrich, hen, turkey. 
$\mathrm{PhD}$ thesis, Agrarian, State University of Moldova.

Samar ME, Avila RE, De Fabro SP, Porfirio V, Esteban FJ, Pedrosa, JA and Peinado MA (1999). Histochemical study of Magellanic Penguin (Spheniscus magellanicus) minor salivary glands during postnatal growth. Anat. Rec. 254 (22), 298-306.

Sayed RK, Abdalla KE, Ahmed AK and Saleh AM (2017). Macro and Microanatomical Studies on the Choanal Slit of Turkey (Meleagris gallopavo). Journal of Advanced Veterinary Research 7(3):75-80.
Stastny K (1985). Birds of Sea and Fresh Water. Hamlyn, London.

Tadjalli M, Mansouri SH and Poostpasand A (2008): Gross anatomy of the oropharynx cavity in the ostrich (Struthio camelus). Iranian Journal of Veterinary Research 9, 316-323.

Tivane C, Rodrigues MN, Soley JT, Groenwaid HB (2011). Gross anatomical features of the oropharyngeal cavity of the ostrich (Struthio camelus). Brazilian Journal of Veterinary Research 31, 543-550. 